\title{
2-absorbing and $n$-weakly prime submodules
}

\author{
S. Moradi and A. Azizi
}




\title{
2-ABSORBING AND $n$-WEAKLY PRIME SUBMODULES
}

\author{
S. MORADI AND A. AZIZI
}

Received 4 May, 2011

\begin{abstract}
Let $R$ be a commutative ring with identity, and let $n>1$ be an integer. A proper submodule $N$ of an $R$-module $M$ will be called 2-absorbing [resp. $n$-weakly prime], if $r, s \in R$ and $x \in M$ with $r s x \in N$ [resp. $r s x \in N \backslash(N: M)^{n-1} N$ ] implies that $r s \in(N: M)$ or $r x \in N$, or $s x \in N$. These concepts are generalizations of the notions of 2-absorbing ideals and weakly prime submodules, which have been studied in $[3,4,6,7]$. We will study 2 -absorbing and $n$ weakly prime submodules in this paper. Among other results, it is proved that if $(N: M)^{n-1} N \neq$ $(N: M)^{2} N$, then $N$ is 2-absorbing if and only if it is $n$-weakly prime.
\end{abstract}

2000 Mathematics Subject Classification: 13A10; 13C99; 13E05; 13F05; 13F15

Keywords: $n$-weakly prime submodule, prime submodule, primary submodule, 2-absorbing submodule, Weakly prime submodule

\section{INTRODUCTION}

Throughout this paper all rings are commutative with identity and all modules are unitary. Also we take $R$ as a commutative ring with identity, $M$ as an $R$-module, and $n>1$ is a positive integer.

Let $N$ be a submodule of $M$. The ideal $\{r \in R \mid r M \subseteq N\}$ is denoted by $(N: M)$.

It is said that a proper submodule $N$ of $M$ is prime if for $r \in R$ and $a \in M$ with $r a \in N$, either $a \in N$ or $r \in(N: M)$. If $N$ is a prime submodule of $M$, then one can easily see that $P=(N: M)$ is a prime ideal of $R$, and we say $N$ is a $P$-prime submodule. Prime submodules have been studied extensively in many papers (see, for example, [2], [4], [3]), so studying its generalization can be helpful in the amplification of this theory.

As a generalization of prime submodules, a proper submodule $N$ of $M$ is called weakly prime, if $r, s \in R$ and $x \in M$ with $r s x \in N$ implies that $r x \in N$ or $s x \in N$ (see $[3,4,7])$.

In this paper, we will introduce and study two generalizations of weakly prime submodules. 


\section{2-ABSORBING SUBMODULES}

According to [6] an ideal $I$ of a ring $R$ is called 2-absorbing, if $a b c \in I$ for $a, b, c \in I$ implies that $a b \in I$ or $b c \in I$ or $a c \in I$.

A generalization of weakly prime submodules, which is also a module version of 2-absorbing ideals, is introduced as follows:

Definition 1. A proper submodule $N$ of $M$ will be called 2-absorbing if for $r, s \in$ $R$ and $x \in M, r s x \in N$ implies that $r s \in(N: M)$ or $r x \in N$ or $s x \in N$.

Lemma 1 (Theorem 2.1, Theorem 2.4, and Theorem 2.5 in [6]). Let I be a 2absorbing ideal of $R$ with $\sqrt{I}=J$. Then

(1) $J$ is a 2-absorbing ideal of $R$ with $J^{2} \subseteq I \subseteq J=\left\{r \in R \mid r^{2} \in I\right\}$.

(2) $\{(I: r)\}_{r \in J \backslash I}$ is a chain of prime ideals.

(3) Either $J$ is a prime ideal of $R$, or $J=P_{1} \cap P_{2}$ with $P_{1} P_{2} \subseteq I$, where $P_{1}, P_{2}$ are the only distinct prime ideals of $R$, which are minimal over $I$.

For each $r \in R$ and every submodule $N$ of $M$, we consider $N_{r}=(N: M r)=\{x \in$ $M \mid r x \in N\}$.

Part (ii) of the following lemma proves that 2-absorbing submodules are not too far from prime submodules.

Proposition 1. Let $N$ be a 2-absorbing submodule of $M$ with $\sqrt{(N: M)}=J$. Then

(i) $(N: M)$ and $J$ are 2-absorbing ideals of $R$. Furthermore $J^{2} \subseteq(N: M) \subseteq J=\left\{r \in R \mid r^{2} \in(N: M)\right\}$.

(ii) If $(\bar{N}: M) \neq J$, then for every $r \in J \backslash(N: M), N_{r}$ is a prime submodule containing $N$ with $J \subseteq\left(N_{r}: M\right)$. Moreover $\left\{\left(N_{r}: M\right)\right\}_{r \in J \backslash(N: M)}$ is a chain of prime ideals.

(iii) Either $J$ is a prime ideal of $R$, or $J=P_{1} \cap P_{2}$, where $P_{1}, P_{2}$ are the only distinct minimal prime ideals over $(N: M)$ and $P_{1} P_{2} \subseteq(N: M)$.

Proof. (i) Let $s, t, r \in R$ with $s t r \in(N: M)$. If $s r, t r \notin(N: M)$, then there exist $x, y \in M \backslash N$ such that $s r x, \operatorname{tr} y \notin N$.

Since $\operatorname{st}(r(x+y)) \in N$ and $N$ is 2-absorbing, $s t \in(N: M)$ or $\operatorname{sr}(x+y) \in N$ or $\operatorname{tr}(x+y) \in N$. If $\operatorname{sr}(x+y) \in N$, then since $s r x \notin N$, we have $\operatorname{sry} \notin N$. So as st $(r y) \in N$ and $\operatorname{tr} y \notin N$, st $\in(N: M)$.

Similarly in case $\operatorname{tr}(x+y) \in N$, we get $s t \in(N: M)$.

Now since $(N: M)$ is a 2-absorbing ideal, by Lemma $1(1), J$ is also a 2-absorbing ideal with $J^{2} \subseteq(N: M) \subseteq J=\left\{r \in R \mid r^{2} \in(N: M)\right\}$.

(ii) To prove that $N_{r}$ is a prime submodule, let $s x \in N_{r}$, where $s \in R \backslash\left(N_{r}: M\right)$ and $x \in M$. Then by the definition of $N_{r}, r s x \in N$ and as $N$ is 2-absorbing, $r s \in(N: M)$ or $r x \in N$ or $s x \in N$.

If $r s \in(N: M)$, then $s r M \subseteq N$, that is $s \in\left(N_{r}: M\right)$, which is a contradiction. If $r x \in N$, then $x \in N_{r}$ by the definition of $N_{r}$, which completes the proof. 
Now suppose $s x \in N$. By part (i), $r^{2} \in J^{2} \subseteq(N: M)$, so $r M \subseteq N_{r}$, particularly $r x \in N_{r}$. Then $(r+s) x \in N_{r}$, that is $r(r+s) x \in N$, and since $N$ is 2-absorbing, $r x \in N$ or $(r+s) x \in N$ or $r(r+s) \in N$.

If $r x \in N$, then $x \in N_{r}$, which completes the proof. Also if $(r+s) x \in N$, then from $s x \in N$, again we get $r x \in N$ and so $x \in N_{r}$.

Now assume $r(r+s) \in(N: M)$. According to part (i), $r^{2} \in J^{2} \subseteq(N: M)$, hence $r s \in(N: M)$, and so $s \in\left(N_{r}: M\right)$. Whence $N_{r}$ is a prime submodule of $M$.

One can easily see that $((N: M): r)=\left(N_{r}: M\right)$. By part (i), $r J \subseteq J^{2} \subseteq(N: M)$, so $J \subseteq((N: M): r)=\left(N_{r}: M\right)$.

For the proof of the rest of this part note that by part (i), $(N: M)$ is a 2-absorbing ideal. Hence by Lemma $1(2),\{((N: M): r)\}_{r \in J \backslash(N: M)}$ is a chain of prime ideals and $\left(N_{r}: M\right)=((N: M): r)$.

(iii) By part (i), $(N: M)$ is a 2-absorbing ideal, so the proof is clear by Lemma $1(3)$.

Let $S$ be a multiplicatively closed subset of $R$, and $W$ a submodule of $S^{-1} M$ as $S^{-1} R$-module. We consider $W^{c}=\left\{x \in M \mid \frac{x}{1} \in W\right\}$.

The proof of the following lemma is easy and we leave it to the reader.

Lemma 2. Let $N$ be an 2-absorbing submodule of $M$, and $S$ a multiplicatively closed subset of $R$.

(i) If $S^{-1} N \neq S^{-1} M$, then $S^{-1} N$ is a 2-absorbing submodule of $S^{-1} M$.

(ii) If $W$ is a 2-absorbing submodule of a $S^{-1} R$-module $S^{-1} M$, then $W^{c}$ is a 2-absorbing submodule of $M$.

Lemma 3 (Proposition 1 in [9]). Let $S$ be a multiplicatively closed subset of $R$. If $N$ is a P-prime submodule of $M$ such that $(N: M) \cap S=\varnothing$, then $S^{-1} N$ is a prime submodule of $S^{-1} M$ as an $S^{-1} R$-module.

Let $N$ be a 2-absorbing submodule of $M$ with $(N: M) \neq \sqrt{(N: M)}$. Then evidently $\left(N_{r}: M\right)=((N: M): r)$, so

according to Proposition 1(ii), $\mathfrak{P}=\cap\{((N: M): r) \mid r \in \sqrt{(N: M)} \backslash(N: M)\}$ is a prime ideal. In this case we say $\mathfrak{P}$ is the prime ideal related to $N$.

Corollary 1. Let $N$ be a 2-absorbing submodule of $M$ with $(N: M) \neq \sqrt{(N: M)}$ and $\operatorname{dim} R<\infty$. Suppose $S$ is a multiplicatively closed subset of $R$, and $\mathfrak{P}$ is the prime ideal related to $N$.

(i) If $S \cap \mathfrak{P}=\varnothing$, then $S^{-1} N$ is a 2-absorbing submodule of $S^{-1} M$.

(ii) $N_{\mathfrak{P}}$ is a 2-absorbing submodule of the $R_{\mathfrak{P}}$-module $M_{\mathfrak{P}}$.

Proof. (i) By Lemma 2(i), it is enough to prove that $S^{-1} M \neq S^{-1} N$. According to Proposition 1(ii), $\left\{\left(N_{r}: M\right)\right\}_{r \in \sqrt{(N: M)} \backslash(N: M)}$ is a chain of prime ideals, and since $\operatorname{dim} R<\infty$, this chain has a minimal element, say $\left(N_{r_{0}}: M\right)$. Now since $\left(N_{r}\right.$ : $M)=((N: M): r)$ for each $r \in \sqrt{(N: M)} \backslash(N: M)$, by our assumption we get 
$S \cap\left(N_{r_{0}}: M\right)=S \cap \mathfrak{P}=\varnothing$. Now according to Proposition 1(ii) and Lemma 3, $S^{-1} N_{r_{0}}$ is a prime submodule of $S^{-1} M$ containing $S^{-1} N$. Hence $S^{-1} N \neq S^{-1} M$.

(ii) The proof is clear by part (i).

Lemma 4. Let $N$ be an $P$-primary submodule of $M$. Then $N$ is 2-absorbing if and only if $P^{2} \subseteq(N: M)$. In particular for every maximal submodule $K$ of $M,(K: M)^{2}$ is a 2-absorbing ideal of $R$.

Proof. If $N$ is 2-absorbing, then by Proposition 1(i), $P^{2} \subseteq(N: M)$.

For the converse suppose that $r s x \in N$ for some $r, s \in R$ and $x \in M$. If $r x, s x \notin N$, then since $N$ is $P$-primary, $r, s \in P$ and so $r s \in P^{2} \subseteq(N: M)$. Therefore $N$ is 2absorbing.

Example 1. Let $\mathfrak{M}$ be a maximal ideal of $R$.

(a) Evidently, every weakly prime submodule is 2-absorbing. In particular if $\left\{P_{i}\right\}_{i \in \mathbb{N}}$ is a chain of prime ideals, then it is easy to see that for the free $R$-module $\oplus_{i \in \mathbb{N}} R$, the submodule $\oplus_{i \in \mathbb{N}} P_{i}$ is 2-absorbing.

(b) Let $F$ be a faithfully flat $R$-module. Then $\mathfrak{M} F$ and $\mathfrak{M}^{2} F$ are 2-absorbing submodules, particularly if $F$ is a free module, or a projective module over an integral domain.

(c) Let $R$ be a Noetherian domain which is not a field. If $F$ is a free $R$-module, then $\mathfrak{M}^{k} F$ is a primary submodule for $2<k \in \mathbb{N}$, but it is not 2-absorbing.

(d) Let $R$ be a Dedekind domain domain which is not a field. If $F$ is a free $R$ module, then $\mathfrak{M}^{2} F$ is a 2-absorbing submodule but it is not weakly prime.

(e) If $R$ is a unique factorization domain and $p$ is an irreducible element of $R$, then for the free $R$-module $R \oplus R$, the submodule $N=R p \oplus R p^{2}$ is 2absorbing, but it is not weakly prime.

Proof. (a) The proof is easy, so it is omitted.

(b) Since $F$ is faithfully flat, $\mathfrak{M} F$ and $\mathfrak{M}^{2} F$ are proper submodules of $F$. Clearly $\sqrt{(N: F)}=\mathfrak{M}$, where $N=\mathfrak{M}^{k} F$ for $k \in \mathbb{N}$. Then $N$ is a primary submodule, since $\sqrt{(N: F)}$ is a maximal ideal. Evidently $\mathfrak{M}^{2} \subseteq\left(\mathfrak{M}^{2} F: F\right)$ and $\mathfrak{M}^{2} \subseteq(\mathfrak{M} F: F)$, so by Lemma 4, the submodules $\mathfrak{M} F$ and $\mathfrak{M}^{2} F$ are 2-absorbing.

(c) It is easy to see that in case $F$ is a free module, $(I F: F)=I$ for each ideal $I$ of $R$. As it was proved in part (b), $\mathfrak{M}^{k} F$ is a primary submodule. However, if $\mathfrak{M}^{k} F$ is 2-absorbing, then $\mathfrak{M}^{2} \subseteq\left(\mathfrak{M}^{k} F: F\right)=\mathfrak{M}^{k} \subseteq \mathfrak{M}^{2}$ according to Lemma 4. Thus $\mathfrak{M}^{2}=\mathfrak{M}^{k}$. Now by Nakayama's lemma, there exists $r \in R$ such that $r \mathfrak{M}^{2}=0$ and $r-1 \in \mathfrak{M}^{k-2}$. Then either $r=0$, or $\mathfrak{M}=0$, and both are impossible.

(d) Note that for every weakly prime submodule $N$ of a module $M$, the ideal $(N: M)$ is prime. Although $\left(\mathfrak{M}^{2} F: F\right)=\mathfrak{M}^{2}$ is not a prime ideal, consequently $\mathfrak{M}^{2} F$ is not weakly prime.

(e) A straightforward calculation shows that $N$ is 2-absorbing. But $N$ is not weakly prime, because $p \cdot p(1,1) \in N$, however $p(1,1) \notin N$. 
Lemma 5 (Lemma 4 in [5]). Let $M$ be a finitely generated $R$-module and $B$ a submodule of $M$. If $(B: M) \subseteq P$, where $P$ is a prime ideal of $R$, then there exists a $P$-prime submodule $N$ of $M$ containing $B$.

Let $P$ be a prime ideal of $R$. For simplification, we denote the submodule $\left(\left(P^{2}\right)_{P} M_{P}\right)^{c}$ of $M$ by $P^{(2)} M$.

The following corollary supplies abundant examples of 2-absorbing submodules.

Corollary 2. Let $P$ be a prime ideal of $R$. If one of the following holds, then $P^{(2)} M$ is 2-absorbing.

(i) $\left(P^{2}\right)_{P} M_{P} \neq M_{P}$.

(ii) $M$ is finitely generated and ann $(M) \subseteq P$.

Proof. (i) Evidently $\left(P^{2}\right)_{P} \subseteq\left(\left(P^{2}\right)_{P} M_{P}: M_{P}\right)$, so $P_{P} \subseteq \sqrt{\left(\left(P^{2}\right)_{P} M_{P}: M_{P}\right)}$, and since $P_{P}$ is a maximal ideal, $\sqrt{\left(\left(P^{2}\right)_{P} M_{P}: M_{P}\right)}=P_{P}$. Therefore $\left(P^{2}\right)_{P} M_{P}$ is a $P_{P}$-primary submodule of $M_{P}$. Then clearly $P^{(2)} M$ is a $P$-primary submodule of $M$. Now the proof is given by Lemma 4, as $P^{2} \subseteq\left(P^{(2)} M: M\right)$.

(ii) By part (i), it is enough to prove that $\left(P^{2}\right)_{P} M_{P} \neq M_{P}$.

According to Lemma 5, there exists a $P$-prime submodule $N$ of $M$. Then by Lemma 3, $N_{P}$ is a $P_{P}$-prime submodule of $M_{P}$. Now from $P_{P} M_{P} \subseteq N_{P}$, we get $\left(P^{2}\right)_{P} M_{P} \subseteq N_{P}$. Consequently $\left(P^{2}\right)_{P} M_{P} \neq M_{P}$.

In the following, if

$\mathcal{A}=\{N \mid N$ is a $P$-primary and 2-absorbing submodule of $M\}=\varnothing$,

then we consider $\bigcap \mathcal{A}=M$.

Corollary 3. If $P$ is a prime ideal of $R$, then

$P^{(2)} M=\bigcap\{N \mid N$ is a $P$-primary and 2-absorbing submodule of $M\}$.

Proof. Set $\mathcal{A}=\{N \mid N$ is a $P$-primary and 2-absorbing submodule of $M\}$.

If $P^{(2)} M=M$, then $\mathcal{A}=\varnothing$, because if $N$ is a $P$-primary and 2-absorbing submodule of $M$, by Lemma $4, P^{2} M \subseteq N$. Therefore $M=P^{(2)} M \subseteq\left(N_{P}\right)^{c}=N$, which is impossible. Hence $\mathcal{A}=\varnothing$, and so in this case $\bigcap \mathcal{A}=M=P^{(2)} M$.

Now let $P^{(2)} M \neq M$. By Corollary 2(i), $P^{(2)} M$ is 2-absorbing. Also in the proof of Corollary 2(i), we showed that $P^{(2)} M$ is $P$-primary, so $P^{(2)} M \in \mathcal{A}$. Consequently $\bigcap \mathcal{A} \subseteq P^{(2)} M$.

Now suppose that $N^{\prime}$ is a $P$-primary and 2-absorbing submodule of $M$. Then Lemma 4 implies that $P^{(2)} M \subseteq\left(N_{P}^{\prime}\right)^{c}=N^{\prime}$. Consequently $P^{(2)} M=\bigcap \mathcal{A}$.

A prime ideal $P$ of $R$ is said to be a divided prime ideal if $P \subseteq R r$ for every $r \in R \backslash P$.

We consider $T(M)=\{m \in M \mid \exists 0 \neq r \in R, r m=0\}$. If $M$ is a nonzero module with $T(M)=0$, then it is easy to see that $R$ is an integral domain, and in this case we say $M$ is a torsion-free module. 
Theorem 1. Let $M$ be a nonzero finitely generated module and $P$ a divided prime ideal. If $T(M) \subseteq P^{2} M$, then $P^{2} M$ is 2-absorbing and

$$
P^{2} M=\bigcap\{N \mid N \text { is a P-primary and 2-absorbing submodule of } M\},
$$

particularly if $M$ is a torsion-free module.

Proof. First we show that $P^{2} M$ is a proper submodule of $M$. If $P^{2} M=M$, then by Nakayama's lemma, there exists $a \in R$ such that $1-a \in P^{2}$ and $a M=0$. Since $1-a \in P, a \notin P$ and as $P$ is a divided prime ideal, $1-a \in P \subseteq R a$. Thus there exists $t \in R$ with $1-a=t a$. Therefore $M=(1-a) M=t a M=0$, which is impossible.

Now by Corollary 3 and Lemma 4 , it suffices to show that $P^{2} M$ is $P$-primary. Suppose that $r x=s_{1} t_{1} y_{1}+\cdots+s_{n} t_{n} y_{n} \in P^{2} M$, where $s_{i}, t_{i} \in P, y_{i}, x \in M$, and $r \in R$. If $r \notin P$, then since $P$ is a divided prime, $P \subseteq R r$, and hence there exist $r_{1}, \ldots, r_{n} \in R$ such that $s_{i}=r r_{i} \in P$, for $i=1, \ldots, n$. Thus for each $i, r_{i} \in P$ and $r\left(r_{1} t_{1} y_{1}+\cdots+r_{n} t_{n} y_{n}\right)=r x \in P^{2} M$. Hence as $x-\left(r_{1} t_{1} y_{1}+\cdots+r_{n} t_{n} y_{n}\right) \in$ $T(M) \subseteq P^{2} M$, and $r_{1} t_{1} y_{1}+\cdots+r_{n} t_{n} y_{n} \in P^{2} M$, we have $x \in P^{2} M$, which completes the proof.

According to [1] an ideal $I$ of $R$ is called an $n$-almost prime ideal if for $a, b \in R$ with $a b \in I \backslash I^{n}$, either $a \in I$ or $b \in I$. The case $n=2$ is called an almost prime ideal and it is due to [8].

Theorem 2. Let $R$ be a Noetherian domain, which is not a field. Then the following are equivalent.

(i) $R$ is Dedekind domain.

(ii) If $I$ is a 2-absorbing ideal of $R$, then $I$ is almost prime or $I=P_{1} \cap P_{2}$ or $I=P^{2}$, where $P, P_{1}, P_{2}$ are prime ideals of $R$.

Proof. (i) $\Rightarrow$ (ii) The proof is given by [6, Theorem 3.14].

(ii) $\Rightarrow$ (i) We prove that every localization of $R$ at any nonzero prime ideal has the property introduced in (ii).

Let $J$ be a 2-absorbing ideal of $R_{\mathfrak{P}}$, where $\mathfrak{P}$ is a nonzero prime ideal of $R$. By Lemma $2, J^{c}$ is a 2-absorbing ideal of $R$, and hence by our assumption, $J^{c}$ is almost prime or $J^{c}=P_{1} \cap P_{2}$ or $J^{c}=P^{2}$, for some prime ideals $P, P_{1}, P_{2}$ of $R$.

By [10, Proposition 2.10(ii)], the localization of an almost prime ideal is almost prime if it is a proper ideal. Hence if $J^{c}$ is an almost prime ideal, then $\left(J^{c}\right)_{\mathfrak{P}}=J \neq$ $R$, and so $J$ is an almost prime ideal of $R_{\mathfrak{P}}$.

If $J^{c}=P_{1} \cap P_{2}$, then $J=\left(J^{c}\right)_{\mathfrak{P}}=\left(P_{1}\right)_{\mathfrak{P}} \cap\left(P_{2}\right)_{\mathfrak{P}}$, and since $J$ is a proper ideal, at least one of $\left(P_{1}\right)_{\mathfrak{P}}$ or $\left(P_{2}\right)_{\mathfrak{P}}$ is a prime ideal. So in this case either $J$ is a prime ideal or the intersection of two prime ideals.

In case $J^{c}=P^{2}$, then $J=\left(J^{c}\right)_{\mathfrak{P}}=\left(P_{\mathfrak{P}}\right)^{2}$, and as $J$ is proper, the ideal $(P)_{\mathfrak{P}}$ is prime. 
Therefore by considering the localization of $R$, we may suppose that $\mathfrak{M}$ is the only maximal ideal of $R$. If $\mathfrak{M}=\mathfrak{M}^{2}$, then by Nakayama's lemma, $\mathfrak{M}=0$, that is $R$ is a field. Now let $s \in \mathfrak{M} \backslash \mathfrak{M}^{2}$, and set $I=\mathfrak{M}^{2}+R s$.

First we prove that every ideal $K$ with $\mathfrak{M}^{2} \subset K$ is almost prime.

Evidently $\sqrt{K}=\mathfrak{M}$, and so $K$ is a primary ideal with $\mathfrak{M}^{2} \subseteq K$. So by Lemma 4, $K$ is 2-absorbing and the hypothesis in (ii) implies that $K$ is almost prime, or $K=P_{1} \cap P_{2}$ or $K=P^{2}$, where $P, P_{1}, P_{2}$ are prime ideals of $R$. If $K=P^{2}$, then $\mathfrak{M}^{2} \subseteq K=P^{2}$, and so $\mathfrak{M}=P$. Thus $K=\mathfrak{M}^{2}$, which is impossible. If $K=P_{1} \cap P_{2}$, then $\mathfrak{M}^{2} \subseteq P_{1}$ and $\mathfrak{M}^{2} \subseteq P_{2}$ and so $P_{1}=P_{2}=\mathfrak{M}$, that is in this case $K=\mathfrak{M}$, so evidently $K$ is (almost) prime.

By $(*)$ in above, $I$ is an almost prime ideal. We will prove that $I^{2}=\mathfrak{M}^{2}$. On the contrary let $a, b \in \mathfrak{M}$ such that $a b \notin I^{2}$. Thus $a b \in I \backslash I^{2}$, and since $I$ is almost prime, we have $a \in I$ or $b \in I$ and not both, as $a b \notin I^{2}$, then suppose $a \in I$ and $b \notin I$. Note that $b^{2} \in \mathfrak{M}^{2} \subseteq I$. Hence $b(a+b) \in I$. If $b(a+b) \notin I^{2}$, then $b \in I$ or $a+b \in I$, which is impossible. Hence $b(a+b) \in I^{2}$, and $a b \notin I^{2}$, therefore $b^{2} \notin I^{2}$. Then $b^{2} \in I \backslash I^{2}$, and so $b \in I$, which is a contradiction.

Consequently $\mathfrak{M}^{2}=I^{2}=\mathfrak{M}^{4}+\mathfrak{M}^{2} s+R s^{2}=\mathfrak{M}^{2}\left(\mathfrak{M}^{2}+R s\right)+R s^{2}$. Hence by Nakayama's lemma $\mathfrak{M}^{2}=R s^{2} \subseteq R s$, and as $s \notin \mathfrak{M}^{2}$, we have $\mathfrak{M}^{2} \subset R s$. Thus again by (*), $R s$ is almost prime. By [8, Lemma 2.6], every principal and almost prime ideal is a prime ideal, hence $R s$ is a prime ideal. Now since $\mathfrak{M}^{2} \subseteq R s, \mathfrak{M}=R s$, that is $\mathfrak{M}$ is a principal ideal. Therefore $R$ is a discrete valuation domain, in case $R$ is local.

Now for the general case, note that every localization of $R$ is a discrete valuation domain, hence $R$ is a Dedekind domain.

\section{3. $n$-WEAKLY PRIME SUBMODULES}

Another generalization of weakly prime submodules is introduced in the following. The following definition is also a generalization and a module version of $n$ almost prime ideals which was introduced and studied in [1].

Definition 2. Let $n>1$ be an integer. A proper submodule $N$ of $M$ will be called $n$-weakly prime, if for $r, s \in R$ and $x \in M, r s x \in N \backslash(N: M)^{n-1} N$ implies that $r s \in(N: M)$ or $r x \in N$ or $s x \in N$.

If we consider $R$ as an $R$-module, then evidently a proper ideal $I$ of $R$ is $n$-weakly prime if for $a, b, c \in R, a b c \in I \backslash I^{n}$ implies that $a b \in I$ or $b c \in I$ or $a c \in I$.

Remark 1. For any submodule, we have the following implications:

(1) Prime $\Longrightarrow$ weakly prime $\Longrightarrow 2$-absorbing $\Longrightarrow \mathrm{n}$-weakly prime.

(2) $n$-weakly prime $\Longrightarrow(n-1)$-weakly prime, for each $n>2$.

Evidently the zero submodule is $n$-weakly prime, but it is not necessarily 2-absorbing. The following example introduces non trivial $n$-weakly prime submodules, which are not 2-absorbing. 
Example 2. Let $R=\frac{K\left[X_{1}, X_{2}, X_{3}, X_{4}\right]}{\left\langle X_{1}{ }^{2}, X_{2}{ }^{2}, X_{3}{ }^{2}, X_{4}{ }^{2}, X_{1} X_{2} X_{3}, X_{1} X_{2} X_{4}, X_{1} X_{3} X_{4}, X_{2} X_{3} X_{4}\right\rangle}$, where $K$ is a field of characteristic 2 and $X_{1}, X_{2}, X_{3}, X_{4}$ are independent indeterminates. Consider $M=R \oplus R$ and $I=\left\langle\bar{X}_{1} \bar{X}_{2}+\bar{X}_{3} \bar{X}_{4}\right\rangle$. Then the two submodules $N=$ $\{(x, x) \mid x \in I\}$ and $N^{\prime}=I \oplus I$ are $n$-weakly prime, but they are not 2-absorbing.

Proof. Evidently $(R, \mathfrak{M})$ is a local ring with $\mathfrak{M}^{3}=0$, where $\mathfrak{M}=\left\langle\bar{X}_{1}, \bar{X}_{2}, \bar{X}_{3}, \bar{X}_{4}\right\rangle$. First we prove that $\bar{X}_{1} \bar{X}_{2}+\bar{X}_{3} \bar{X}_{4}$ is irreducible.

Suppose $f g=\bar{X}_{1} \bar{X}_{2}+\bar{X}_{3} \bar{X}_{4} \quad(*)$, with $f, g$ non unit. Note that $\mathfrak{M}^{3}=0$, then we can consider $f=a_{1} \bar{X}_{1}+a_{2} \bar{X}_{2}+a_{3} \bar{X}_{3}+a_{4} \bar{X}_{4} \in \mathfrak{M}$, and $g=b_{1} \bar{X}_{1}+b_{2} \bar{X}_{2}+$ $b_{3} \bar{X}_{3}+b_{4} \bar{X}_{4} \in \mathfrak{M}$, where $a_{i}, b_{i} \in K$. From (*) we get:
(1) $a_{1} b_{2}+a_{2} b_{1}=1$
(2) $a_{1} b_{3}+a_{3} b_{1}=0$
(3) $a_{1} b_{4}+a_{4} b_{1}=0$
(4) $a_{2} b_{3}+a_{3} b_{2}=0$
(5) $a_{2} b_{4}+a_{4} b_{2}=0$
(6) $a_{3} b_{4}+a_{4} b_{3}=1$

By (2), $\circ=a_{1} b_{4}\left(a_{1} b_{3}+a_{3} b_{1}\right)$ and by (3), $\circ=a_{1} b_{3}\left(a_{1} b_{4}+a_{4} b_{1}\right)$ and so $a_{1} b_{1}\left(a_{3} b_{4}-a_{4} b_{3}\right)=0$. Since the characteristic of $K$ is $2,-a_{4} b_{3}=a_{4} b_{3}$ and so $a_{1} b_{1}\left(a_{3} b_{4}+a_{4} b_{3}\right)=0$. Hence by (6), $a_{1} b_{1}=0$. Then $a_{1}=\circ$ or $b_{1}=0$. The case $a_{1}=b_{1}=\circ$ is impossible, by (1). If $\circ=a_{1}$ and $\circ \neq b_{1}$, then (2) and (3) imply that $a_{3}=\circ=a_{4}$ and this is a contradiction by (6).

In case $\circ \neq a_{1}$ and $\circ=b_{1}$, then by (2), (3) we get $b_{3}=\circ=b_{4}$, which is a again impossible, according to (6). Consequently $\bar{X}_{1} \bar{X}_{2}+\bar{X}_{3} \bar{X}_{4}$ is irreducible.

One can easily see that $(N: M)=0$, and so $(N: M)^{n-1} N=0$. Also it is easy to see that $I \subseteq \mathfrak{M}^{2}$ and $\left(N^{\prime}: M\right)=I$. Then $I^{2} \subseteq \mathfrak{M}^{4}=0$, and thus $\left(N^{\prime}: M\right)^{n-1} N^{\prime}=$ 0 .

To show that $N$ is $n$-weakly prime, let $(\circ, \circ) \neq r s(a, b) \in N$, where $r, s \in R$ and $(a, b) \in M$. We can assume $\circ \neq r s a \in I$. Then for some $h \in R, \circ \neq r s a=h\left(\bar{X}_{1} \bar{X}_{2}+\right.$ $\left.\bar{X}_{3} \bar{X}_{4}\right)$. But since $I \mathfrak{M} \subseteq \mathfrak{M}^{3}=0, h \in R \backslash \mathfrak{M}$. Thus $h$ is unit and so $r s a h^{-1}=$ $\bar{X}_{1} \bar{X}_{2}+\bar{X}_{3} \bar{X}_{4}$ and it is irreducible, therefore $r$ or $s a$ is unit. Hence $r$ or $s$ is unit and so $s(a, b) \in r^{-1} N=N$ or $r(a, b) \in s^{-1} N=N$. This show that $N$ is $n$-weakly prime. The same argument proves that $N^{\prime}$ is $n$-weakly prime.

Now if on the contrary $N$ is a $2-$ absorbing submodule, then again by Proposition 1(i), $(N: M)=0$ must be a $2-$ absorbing ideal and as $0=\mathfrak{M}^{3} \subseteq(N: M)$, we will have $\mathfrak{M}^{2} \subseteq(N: M)=0$, which is impossible. Thus $N$ is not a 2-absorbing submodule.

If $N^{\prime}$ is a $2-$ absorbing submodule, then by Proposition 1(i), $\left(N^{\prime}: M\right)=I$ is a 2-absorbing ideal of $R$ and since $0=\mathfrak{M}^{3} \subseteq I$, then $\mathfrak{M}^{2} \subseteq I$. Consequently $\bar{X}_{1} \bar{X}_{2} \in$ $\mathfrak{M}^{2} \subseteq I$. Then for some $h^{\prime} \in R, \circ \neq \bar{X}_{1} \bar{X}_{2}=h^{\prime}\left(\bar{X}_{1} \bar{X}_{2}+\bar{X}_{3} \bar{X}_{4}\right)$. As $\mathfrak{M}^{3}=0, h^{\prime}$ is unit and since $\bar{X}_{1} \bar{X}_{2}+\bar{X}_{3} \bar{X}_{4}$ is irreducible, $\bar{X}_{1}$ or $\bar{X}_{2}$ is unit, which is impossible.

Evidently $(N: M)^{n-1} N \subseteq(N: M)^{2} N$, for each submodule $N$ of $M$ for each $n>2$. We now introduce a simple criteria for an $n$-weakly prime submodule to be 2-absorbing. 
Theorem 3. Let $N$ be a submodule of $M$ with $(N: M)^{2} N \nsubseteq(N: M)^{n-1} N$. Then $N$ is 2-absorbing if and only if it is n-weakly prime.

Proof. Let $N$ be an $n$-weakly prime submodule. Suppose $r s x \in N$, where $r, s \in R$ and $x \in M$. If $r x, s x \notin N$ and $r s \notin(N: M)$, then we prove that $(N: M)^{2} N \subseteq(N$ : $M)^{n-1} N$, which is impossible and so $N$ is 2-absorbing.

First we show that the following facts hold:

(i) $r s x \in(N: M)^{n-1} N$.

(ii) $r s N \subseteq(N: M)^{n-1} N$.

(iii) $r(N: M) x, s(N: M) x \subseteq(N: M)^{n-1} N$.

(iv) $(N: M)^{2} x \subseteq(N: M)^{n-1} N$.

(v) $r(N: M) N, s(N: M) N \subseteq(N: M)^{n-1} N$.

(i) Since $N$ is $n$-weakly prime and $r x, s x \notin N$ and $r s \notin(N: M)$, then $r s x \in(N$ : $M)^{n-1} N$.

(ii) If $r s N \nsubseteq(N: M)^{n-1} N$, then for some $y \in N$ we have $r$ sy $\notin(N: M)^{n-1} N$. So since $r s x \in(N: M)^{n-1} N, r s(x+y) \notin(N: M)^{n-1} N$. Hence $r s(x+y) \in N \backslash(N$ : $M)^{n-1} N$ and then $r(x+y) \in N$ or $s(x+y) \in N$ or $r s \in(N: M)$. Thus $r x \in N$ or $s x \in N$ or $r s \in(N: M)$, which is impossible. Consequently $r s N \subseteq(N: M)^{n-1} N$.

(iii) Let $r(N: M) x \nsubseteq(N: M)^{n-1} N$. Then there exists $t \in(N: M)$ such that $r t x \in N \backslash(N: M)^{n-1} N$. Clearly $r(s+t) x \in N$. We have $r(s+t) x \notin(N: M)^{n-1} N$, otherwise since $r s x \in(N: M)^{n-1} N$, rtx $\in(N: M)^{n-1} N$, which is a contradiction. Then $r(s+t) x \in N \backslash(N: M)^{n-1} N$ and hence $r x \in N$ or $(s+t) x \in N$ or $r(s+t) \in$ $(N: M)$, which implies $r x \in N$ or $s x \in N$ or $r s \in(N: M)$, a contradiction to our assumption. Therefore $r(N: M) x \subseteq(N: M)^{n-1} N$. Similarly $s(N: M) x \subseteq(N$ : $M)^{n-1} N$.

(iv) Let $a, b \in(N: M)$. If $a b x \notin(N: M)^{n-1} N$, then since $r s x \in N,(a+r)(b+$ $s) x \in N$. we show that $(a+r)(b+s) x \notin(N: M)^{n-1} N$.

If $(a+r)(b+s) x \in(N: M)^{n-1} N$, then $r s x+r b x+a s x+a b x \in(N: M)^{n-1} N$, and so by parts (i), (iii), $r s x+r b x+a s x \in(N: M)^{n-1} N$. Hence $a b x \in(N$ : $M)^{n-1} N$, which is impossible. Thus $(a+r)(b+s) x \notin(N: M)^{n-1} N$. Therefore $(a+r)(b+s) x \in N \backslash(N: M)^{n-1} N$ and so $(a+r) x \in N$ or $(b+s) x \in N$ or $(a+r)(b+s) \in(N: M)$, which implies $r x \in N$ or $s x \in N$ or $r s \in(N: M)$, and this is a contradiction. Then $a b x \in(N: M)^{n-1} N$ and so $(N: M)^{2} x \subseteq(N: M)^{n-1} N$.

(v) If for some $b \in(N: M)$ and $y \in N$, rby $\notin(N: M)^{n-1} N$, then $r(s+b)(x+$ $y) \in N$. By parts (i),(ii),(iii), $r s x+r s y+r b x \in(N: M)^{n-1} N$ and since $r b y \notin$ $(N: M)^{n-1} N$, then $r(s+b)(x+y) \notin(N: M)^{n-1} N$. Hence $r(x+y) \in N$ or $(s+$ $b)(x+y) \in N$ or $r(s+b) \in(N: M)$. Then $r x \in N$ or $s x \in N$ or $r s \in(N: M)$, which is a contradiction. Consequently $r(N: M) N \subseteq(N: M)^{n-1} N$ and similarity $s(N: M) N \subseteq(N: M)^{n-1} N$.

Now we prove the theorem. Let $a, b \in(N: M)$ and $y \in N$. If $a b y \notin(N: M)^{n-1} N$, then obviously $(a+r)(b+s)(x+y) \in N$. If $(a+r)(b+s)(x+y) \in(N: M)^{n-1} N$, then by previous parts $a b y=(a+r)(b+s)(x+y)-(a b x+a s x+a s y+r b x+$ 
$r b y+r s x+r s y) \in(N: M)^{n-1} N$, which is impossible. Thus $(a+r)(b+s)(x+$ $y) \notin(N: M)^{n-1} N$ and so $(a+r)(b+s)(x+y) \in N \backslash(N: M)^{n-1} N$. Hence $(a+$ $r)(x+y) \in N$ or $(b+s)(x+y) \in N$ or $(a+r)(b+s) \in(N: M)$. Therefore $r x \in N$ or $s x \in N$ or $r s \in(N: M)$, which is impossible. Consequently $(N: M)^{2} N \subseteq(N$ : $M)^{n-1} N$.

Corollary 4. Let $n>3$ and $M$ be a nonzero torsion-free Noetherian $R$-module. Then a submodule is 2-absorbing if and only if it is $n$-weakly prime.

Proof. Let $N$ be an $n$-weakly prime submodule. By Theorem 3, it is enough to prove that $(N: M)^{n-1} N \neq(N: M)^{2} N$. On the contrary suppose that $(N: M)^{n-1} N=(N: M)^{2} N$. Then by Nakayama's lemma there exists $a \in(N$ : $M)^{n-3}$ such that $(a-1)(N: M)^{2} N=0$. As $M$ is torsion-free, we have $a=1$, or $(N: M)=0$ or $N=0$.

If $a=1$, then $N=M$, which is impossible. Evidently $N=0$ is 2-absorbing. Now suppose $(N: M)=0$. Assume $r s x \in N$, where $r, s \in R$ and $x \in M$. If $r s x \neq 0$, then $r s x \in N \backslash(N: M)^{n-1} N$, and since $N$ is $n$-weakly prime, the proof is clear in this case.

In case $r s x=0$, then $r s=0 \in(N: M)$, or $x=0 \in N$.

Proposition 2. Let $x \in M$ and $a \in R$.

(i) If ann $n_{M}(a) \subseteq a M$, then the submodule $a M$ is 2-absorbing if and only if it is $n$-weakly prime.

(ii) If ann $n_{R}(x) \subseteq(R x: M)$, then the submodule $R x$ is 2-absorbing if and only if $R x$ is $n$-weakly prime.

Proof. (i) Let $M$ be an $n$-weakly prime submodule and $r, s \in R$ and $x \in M$ with $r s x \in a M$. If $r s x \notin(a M: M)^{n-1} a M$, then $r s \in(a M: M)$ or $r x \in a M$ or $s x \in a M$. Therefore assume $r s x \in(a M: M)^{n-1} a M$. Clearly $r(s+a) x=r s x+\operatorname{rax} \in a M$. If $r(s+a) x \notin(a M: M)^{n-1} a M$, then $r(s+a) \in(a M: M)$ or $r x \in a M$ or $(s+a) x \in$ $a M$. So as $a \in(a M: M)$, $r s \in(a M: M)$ or $r x \in a M$ or $s x \in a M$.

Now suppose that $r(s+a) x \in(a M: M)^{n-1} a M$. Then since $r s x \in(a M$ : $M)^{n-1} a M$, for some $y \in(a M: M)^{n-1} M$, we have $a r x=a y$ and so $a(r x-y)=0$. Hence $r x-y \in a \operatorname{ann}_{M}(a) \subseteq a M$ and $y \in(a M: M)^{n-1} M=(a M: M)^{n-2}(a M$ : $M) M \subseteq a M$. Thus $r x \in a M$.

(ii) Let $R x$ be an $n$-weakly prime submodule and $r, s \in R, y \in M$ with $r s y \in R x$. Since $R x$ is $n$-weakly prime, we may assume $r s y \in(R x: M)^{n-1} R x$. Evidently $r s(x+y) \in R x$. If $r s(x+y) \notin(R x: M)^{n-1} R x$, then $r s \in(R x: M)$ or $r(x+y) \in$ $R x$ or $s(x+y) \in R x$. Hence $r s \in(R x: M)$ or $r y \in R x$ or $s y \in R x$.

Now let $r s(x+y) \in(R x: M)^{n-1} R x$. Then as $r s y \in(R x: M)^{n-1} R x, r s x \in$ $(R x: M)^{n-1} R x$ and so $r s x=t x$, for some $t \in(R x: M)^{n-1} \subseteq(R x: M)$. Hence $r s-t \in \operatorname{ann}(x) \subseteq(R x: M)$ and thus $r s \in(R x: M)$.

Example 3. Let $R$ be a unique factorization domain, $p$ an irreducible element of $R$, and $M=R \oplus R$. 
(a) The submodule $N=p^{2} M$ is 2-absorbing.

(b) The submodule $N=p^{3} M$ is neither 2-absorbing, nor 2-weakly prime.

Proof. (a) Consider $a b(c, d) \in N$, where $a, b, c, d \in R$. Then a straightforward calculation shows that $a(c, d) \in N$ or $b(c, d) \in N$ or $p^{2} \mid a b$.

(b) If $N$ is 2-absorbing, then by Proposition 1(i), $(N: M)$ is 2-absorbing and evidently $p^{3} \in(N: M)$, therefore $p^{2} \in(N: M)$. Then $p^{2}(1,0) \in N=p^{3} M$. Hence there exists $t \in R$ with $p^{2}=p^{3} t$. Then $p t=1$, which is impossible. Therefore $N$ is not 2-absorbing and by Proposition 2(i), $N$ is not 2-weakly prime.

Recall that the set of zero divisors of $M$, denoted by $Z(M)$ is defined by $Z(M)=$ $\{r \in R \mid \exists 0 \neq x \in M, r x=0\}$.

The following result studies the behavior of $n$-weakly prime submodules under localization. Its proof is not difficult and we leave it to the reader.

Proposition 3. Let $S$ be a multiplicatively closed subset of $R$.

(i) If $N$ is an $n$-weakly prime submodule of $M$ with $S^{-1} N \neq S^{-1} M$, then $S^{-1} N$ is an $n$-weakly prime submodule of $S^{-1} M$.

(ii) Let $N$ be an $n$-weakly prime submodule of $M$ with $Z\left(\frac{M}{N}\right) \cap S=\varnothing$. Then $S^{-1} N$ is an n-weakly prime submodule of $S^{-1} M$ and $\left(S^{-1} N\right)^{c}=N$. Moreover $S^{-1}(N: M)=\left(S^{-1} N: S^{-1} M\right)$.

We can introduce the concept of $n$-weak prime as follows:

A proper submodule $N$ of $M$ will be called n-weakly prime, if for $r, s \in R$ and $x \in M, r s x \in N \backslash(N: M)^{n-1} N$ implies that $r x \in N$ or $s x \in N$.

Then similar to the proof of Theorem 3, Corollary 4 and Proposition 2 we can prove the following results:

(1) Let $N$ be a submodule of $M$ with $(N: M)^{2} N \nsubseteq(N: M)^{n-1} N$. Then $N$ is weakly prime if and only if it is $n$-weak prime.

(2) Let $n>3$ and $M$ be a nonzero torsion-free Noetherian $R$-module. Then a submodule is weakly prime if and only if it is $n$-weak prime.

(3) Let $a \in R$ with $a n n_{M}(a) \subseteq a M$. Then $a M$ is a weakly prime if and only if it is $n$-weak prime.

\section{REFERENCES}

[1] D. D. Anderson and M. Bataineh, "Generalizations of prime ideals," Commun. Algebra, vol. 36, no. 2, pp. 686-696, 2008.

[2] A. Azizi, "Intersection of prime submodules and dimension of modules," Acta Math. Sci., Ser. B, Engl. Ed., vol. 25, no. 3, pp. 385-394, 2005.

[3] A. Azizi, "Weakly prime submodules and prime submodules," Glasg. Math. J., vol. 48, no. 2, pp. 343-346, 2006.

[4] A. Azizi, "On prime and weakly prime submodules," Vietnam J. Math., vol. 36, no. 3, pp. 315-325, 2008.

[5] A. Azizi and H. Sharif, "On prime submodules," Honam Math. J., vol. 21, no. 1, pp. 1-12, 1999. 
[6] A. Badawi, "On 2-absorbing ideals of commutative rings," Bull. Aust. Math. Soc., vol. 75, no. 3, pp. 417-429, 2007.

[7] M. Behboodi and H. Koohy, "Weakly prime modules," Vietnam J. Math., vol. 32, no. 2, pp. 185 195, 2004.

[8] S. M. Bhatwadekar and P. K. Sharma, "Unique factorization and birth of almost primes," Commun. Algebra, vol. 33, no. 1, pp. 43-49, 2005.

[9] C.-P. Lu, "Spectra of modules," Commun. Algebra, vol. 23, no. 10, pp. 3741-3752, 1995.

[10] S. Moradi and A. Azizi, "Generalizations of prime submodules," vol. preprint.

\section{Authors' addresses}

\section{S. Moradi}

Department of Mathematics, College of Sciences, Shiraz University, Shiraz, 71457-44776, Iran

A. Azizi

Department of Mathematics, College of Sciences, Shiraz University, Shiraz, 71457-44776, Iran

E-mail address: aazizi@shirazu.ac.ir, a_azizi@yahoo.com 